\title{
Toward understanding the genetic basis of adaptation to high-elevation life in poikilothermic species: A comparative transcriptomic analysis of two ranid frogs, Rana chensinensis and R. kukunoris
}

Weizhao Yang ${ }^{1,2}$, Yin $\mathrm{Qi}^{1}, \mathrm{Ke} \mathrm{Bi}^{3}$ and Jinzhong $\mathrm{Fu}^{1,4^{*}}$

\begin{abstract}
Background: Understanding how organisms adapt to high-elevation environments at a genome scale provides novel insights into the process of adaptive evolution. Previous studies have mainly focused on endothermic organisms, while poikilothermic species may have evolved different mechanisms to cope with high-elevation environments. In this context, we sequenced transcriptomes of a pair of closely related anuran species, Rana chensinensis and $R$. kukunoris, which inhabit respective low- and high-elevation habitats. By comparing the two transcriptomes, we identified candidate genes that may be involved in high-elevation adaption in poikilothermic species.

Results: Over 66 million sequence reads from each transcriptome were generated. A total of 41,858 and 39,293 transcripts for each species were obtained by de novo assembly. By comparing the orthologous transcripts, we identified 125 protein-coding genes that have likely experienced strong positive selection (Ka/Ks $>1)$. In addition, 335 genes that may bear a signature of positive selection ( $1 \geq \mathrm{Ka} / \mathrm{Ks}>0.5)$ were also recognized. By considering their functions, fourteen candidate genes were determined to be likely involved in high-elevation adaptation, including two CYP genes, USP-1, and several others.

Conclusions: We identified a set of candidate genes that may have promoted adaptation of $R$. kukunoris to its high-elevation environment. These include several genes that have previously been associated with oxygen binding, response to UV radiation, and repair of free radical injury. Detailed molecular, physiological, and phenotypic studies are required to better understand the roles of these genes in improving the performance of R. kukunoris in a high-elevation environment. We have evidence for both convergent and divergent evolution between endothermic and poikilothemic species, but additional research across a wide range of organisms will be necessary to comprehend the complexity of high-elevation adaptation.
\end{abstract}

Keywords: Adaptation, High elevation, Transcriptome, Amphibian, Positive selection, Ka/Ks ratio, Candidate gene, Oxygen binding, UV radiation

\footnotetext{
* Correspondence: jfu@uoguelph.ca

'Chengdu Institute of Biology, Chinese Academy of Sciences, Chengdu 610041, China

${ }^{4}$ Department of Integrative Biology, University of Guelph, Guelph, Ontario N1G 2W1, Canada

Full list of author information is available at the end of the article
} 


\section{Background}

Elucidating the process of adaptation and understanding its genetic basis is a major mission of modern evolutionary biology $[1,2]$. Although there are a few genome-level studies of model organisms [3,4], most previous studies have focused on a single or a few candidate genes, which limited our understanding of the molecular basis of adaptation in many non-model systems. Recent advances in DNA sequencing technology and bioinformatic tools enable us to access and analyse massive sequence data in a short time at an affordable cost, which allows largescale comparisons at genome, exome, or transcriptome levels [5,6]. These advances present new opportunities for examining the genetic basis of many adaptive processes.

Understanding how organisms adapt to high-elevation environments can provide important insights into the process of adaptive evolution, particularly interactions and trade-offs between genes and pathways involved in simultaneous adaptive responses to multiple environmental challenges [7-10]. High-elevation environments impose severe physiological challenges to organisms, particularly those related to declined levels of oxygen, low temperature, and elevated levels of ultraviolet (UV) radiation $[11,12]$. Physiological variation and adaptation to high-elevation habitats are well documented in many vertebrates [13-15]; however, study of their genetic basis is still in its infancy. Much of the previous work has focused on a few candidate loci, such as hemoglobin related genes and mitochondrial genes [16-18]. Only recently, analyses of genome-wide variations among species and populations living in different altitudinal environments have begun to shed light into the genetic basis of many adaptive processes. For example, genes related to hypoxia response and energy metabolism were shown to be responsible for physiological adaptations in several high-elevation species [9,10,19-21]. All these genome-wide studies investigated endothermic species. Ectothermic species have large physiological differences and may have evolved different mechanisms for adaption to high-elevation environments [12]. For example, instead of maintaining high levels of metabolism for temperature stability, poikilothermic species may lower their metabolic rate to cope with low external temperatures [12]. Understanding the genetic basis of adaptation to high elevations in poikilothermic species will not only complement our knowledge about endothermic species, but may also reveal novel mechanisms that are applicable to species that represent a much larger portion of the tree of life.

A pair of anuran species, Rana chensinensis and $R$. kukunoris, provide an excellent model system to study how poikilothermic species adapt to high-altitude habitats. Rana chensinensis is a widespread species and primarily occurs at low elevations of 600-1300 m above sea level (a.s.l.). Rana kukunoris is a high-elevation species that is only found at 2800-4000 m a.s.l. [22]. Previous studies suggest that the two species descended from a $R$. chensinensis-like common ancestor that inhabited low elevations, and speciation of $R$. kukunoris coincided with the recent uplift of the Tibetan Plateau approximately 7.8 million years ago [23]. Both species are diploid with 24 pairs of chromosomes [24] and $R$. chensiensis has a diploid genome size of 10.05 picograms (approximately 9,800 mega base pairs) [25].

The objective of this study is to make a genome wide search for genes that may be involved in adaptation to high-elevation environments in these amphibians, and identify their associated functions. We sampled multiple tissues of $R$. chensinensis and $R$. kukunoris and sequenced their transcriptomes using an Illumina sequencing platform. By comparing their transcriptomes, we describe genes showing strong signals of positive selection. The functional and phenotypic outcomes of these candidate genes are further inferred by annotating to genomic resources of a variety of model vertebrate species. We report a list of candidate genes that are highly likely to be involved in high-elevation adaptation processes.

\section{Results and discussion}

\section{Illumina sequencing, de novo assembly, and gene} annotation

A total of $67,676,712$ sequence reads of $R$. chensinensis, and $66,476,534$ sequence reads of $R$. kukunoris, were generated by Illumina sequencing. We first filtered these reads, and removed 527,878 and 711,299 defective reads for each species, respectively.

De novo assembly of cleaned reads was performed using various combinations of $\mathrm{K}$-mer lengths and coverage cut-off values [26,27]. In total, 30 raw assemblies were obtained for each species and were further merged by integrating sequence overlaps and eliminating redundancies for each species. The total length of the final set of assemblies was 38.1 mega base pairs (Mb) for $R$. chensinensis and $37.6 \mathrm{Mb}$ for $R$. kukunoris. For $R$. chensinensis, 41,858 transcripts were obtained with an N50 of 1,333 base pairs (bp) and a mean length of $909 \mathrm{bp}$, and for R. kukunoris, 39,293 transcripts were obtained with an N50 of 1,485 bp and a mean length of $956 \mathrm{bp}$. All information of data production is summarized in Table 1, and the length distribution of all transcripts is shown in Figure 1.

We constructed a reference dataset using combined protein data from seven species that represented all major lineages of vertebrates for gene annotation. Transcripts that did not match the reference dataset were blasted against a non-redundant (NR) protein 
Table 1 Summary of transcriptome data for Rana chensinensis and R. kukunoris

\begin{tabular}{|c|c|c|c|c|c|}
\hline & \multicolumn{2}{|c|}{ R. chensinensis } & & \multicolumn{2}{|c|}{ R. kukunoris } \\
\hline & raw assemblies & final assembly & & raw assemblies & final assembly \\
\hline Total number of reads & \multicolumn{2}{|c|}{$67,676,712$} & & \multicolumn{2}{|c|}{$66,476,534$} \\
\hline Read length (bp) & \multicolumn{2}{|c|}{90} & & \multicolumn{2}{|c|}{90} \\
\hline Total length of reads (bp) & \multicolumn{2}{|c|}{$6,090,904,080$} & & \multicolumn{2}{|c|}{$5,982,888,060$} \\
\hline Total length of assembly (Mb) & 742.5 & 38.1 & & 751.4 & 37.6 \\
\hline Total sequences of assembly & $1,624,822$ & 41,858 & & $1,521,607$ & 39,293 \\
\hline N50 length of assembly (bp) & 515 & 1,333 & & 586 & 1,485 \\
\hline Mean length of assembly(bp) & 456 & 909 & & 493 & 956 \\
\hline Median length of assembly(bp) & 325 & 599 & & 340 & 601 \\
\hline Total number of transcripts & \multicolumn{2}{|c|}{41,858} & & \multicolumn{2}{|c|}{39,293} \\
\hline Transcript annotated & \multicolumn{2}{|c|}{16,738} & & \multicolumn{2}{|c|}{16,549} \\
\hline Number of unique genes represented & \multicolumn{2}{|c|}{11,179} & & \multicolumn{2}{|c|}{11,140} \\
\hline Number of putative orthologs & & & 7069 & & \\
\hline
\end{tabular}

$\mathrm{bp}=$ base pair; $\mathrm{Mb}=$ mega base pairs.

database of the GenBank. In total, 16,738 transcripts of $R$. chensinensis were annotated to 11,179 unique genes, accounting for $40.0 \%$ of the total transcripts. Some transcripts were likely products of alternative splicing. The E-value distribution showed that $55.3 \%$ of the annotated transcripts had an E-value below 1E-50, and the similarity distribution showed that $59.2 \%$ of the annotated transcripts had a similarity greater than $60 \%$. Similarly, 16,549 transcripts of $R$. kukunoris were annotated to 11,140 unique genes, accounting for $42.1 \%$ of the total transcripts. Furthermore, 56.6\% of the annotated transcripts had an E-value below 1E-50, and $59.7 \%$ of the annotated transcripts had a similarity greater than 60\% (Table 1 and Additional file 1).

In agreement with previous studies, we found that the quality of sequence reads and appropriate assembly methods are essential in obtaining a reliable de novo assembly, which is the foundation of all other analyses $[26,28,29]$. In particular, de novo assembly by exploring a wide range of K-mer and cut-off values is a powerful

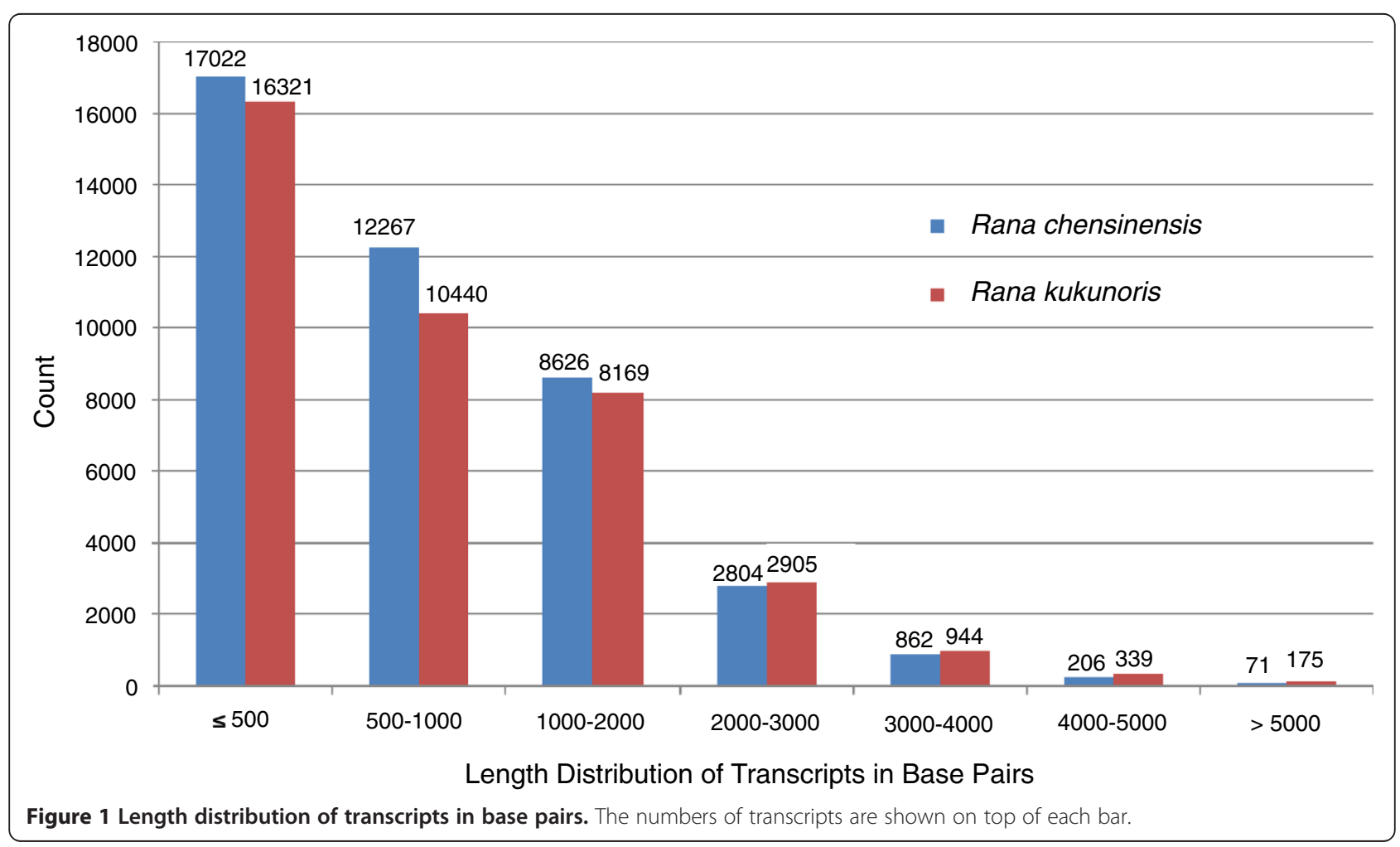


approach. Due to the differential gene expression, sequence coverage among transcripts tends to be uneven, which poses various challenges for de novo assembly $[26,27]$. The use of high K-mer lengths can generate more contiguous assemblies of highly expressed transcripts. On the other hand, the use of low K-mer lengths likely helps to obtain better assemblies for under expressed genes [26]. Adjusting coverage cut-off value can also help to increase the assembly quality when there are similar regions among homologous transcripts [27]. We used five different K-mer lengths and six different coverage cut-off values. In both species, the final assembly had larger N50 and mean lengths than any of the raw assemblies generated by using a single value of both parameters (Table 1). The final assembly also had a higher proportion of annotated transcripts ( 40\%) than all raw assemblies. Moreover, a final step that maps all reads back to the assembled transcripts can also help to improve the quality of assembly. This process allows for a revision of any potential assembly errors and ascertains the consensus sequences representing the majority base calls at heterozygous sites.

\section{Gene ontology (GO) classification}

Gene Ontology category was widely used to classify gene functions [30]. We first removed 5,559 transcripts for $R$. chensinensis and 5,409 transcripts for $R$. kukunoris that were likely generated from alternative splicing. Among the remaining 11,179 transcripts of $R$. chensinensis, which likely represented the same number of unique genes, a total of 8,781 transcripts had the GO annotation under three main divisions. Likewise, among the 11,140 annotated transcripts of $R$. kukunoris, 8,806 transcripts had the GO annotation. The GO category distributions of the transcripts for both species were highly similar (Additional file 2). Within the function of "Cellular Component", 13 level-2 categories were identified, and the terms "cell", "cell part", and "organelle" were the dominant components $(>50 \%)$. Within the function of "Molecular Functions", 15 level-2 categories were identified, and the term "binding" was dominant (>50\%). Within the third function of "Biological Process", 23 level-2 categories were identified, and the terms "cellular process", "metabolic process", "biological regulation", and "pigmentation" were the main functional categories (>50\%).

\section{Identification of putative orthologs}

Reciprocal best hit method was used to identify putative orthologs between the two species [31]. A total of 10,132 pairs of putative orthologs were identified by comparing all annotated transcripts. We then compared each pair of putative orthologs to the reference to determine their open reading frames (ORF) and eliminated the sequences with unexpected stop-codons and ambiguous alignments. After filtering the redundant orthologs that may result from alternative splicing, 7,069 pairs of unique orthologs were finally identified and used in the downstream analyses.

We also identified 8,393 pairs of possible orthologs from transcripts that could not be annotated. We were able to use EBOSS-GETORF [32] to predict the ORFs of 3,880 and 3,851 transcripts in $R$. chensinensis and $R$. kukunoris, respectively. Among these transcripts, 1,701 pairs were properly aligned, which were also used in the test for positive selection.

\section{Test for positive selection}

The $\mathrm{Ka} / \mathrm{Ks}$ ratio was used to test for positive selection [33]. Among the 7,069 pairs of annotated orthologs, 724 pairs were identical, 2,827 pairs had only synonymous or non-synonymous substitutions, and 3,518 pairs had both types of substitutions, for which the $\mathrm{Ka} / \mathrm{Ks}$ ratio were calculated. The mean values of $\mathrm{Ka}, \mathrm{Ks}$, and $\mathrm{Ka} / \mathrm{Ks}$ ratio of all orthologous pairs were $0.0054,0.0372$, and 0.1452 , respectively.

A total of 125 (1.8\%) pairs of orthologs had a $\mathrm{Ka} / \mathrm{Ks}$ ratio greater than 1 , indicating these genes may have experienced strong positive selection (Figure 2). In addition, a $\mathrm{Ka} / \mathrm{Ks}$ ratio of 0.5 was also suggested as a useful cut-off value to identify genes under positive selection [34], and in our study, 335 (4.7\%) pairs of orthologs had a $\mathrm{Ka} / \mathrm{Ks}$ ratio between 0.5 and 1 (Figure 2). Therefore, all these 460 orthologs $(\mathrm{Ka} / \mathrm{Ks}>0.5)$ were considered as candidate genes that have likely experienced positive selection.

Among the 1,701 pairs of predicted coding sequences from un-annotated transcripts, $25(1.5 \%)$ pairs had a $\mathrm{Ka} /$ Ks ratio greater than 1 and $205(12.0 \%)$ pairs had a Ka/ Ks ratio between 0.5 and 1 . They were supplementary to the annotated candidate gene list for future studies.

We clustered all the unique orthologous transcripts to GO categories. Among the 7,069 annotated unique orthologs, 6,811 were assigned to $8,335 \mathrm{GO}$ terms, among which 1,143 terms had at least 10 orthologs. The mean $\mathrm{Ka} / \mathrm{Ks}$ ratio for each of the 1,143 terms was calculated (Additional file 3), and 330 (28.9\%) terms had a mean $\mathrm{Ka} /$ Ks ratio higher than the mean ratio of all orthologs (0.1452). The terms with the highest $\mathrm{Ka} / \mathrm{Ks}$ ratio included "adult heart development", "sarcomere organization" and "condensed nuclear chromosome". Likewise, the terms with the smallest $\mathrm{Ka} / \mathrm{Ks}$ ratio included "protein $\mathrm{K} 63$ linked ubiquitination", "protein K48-linked ubiquitination" and "ubiquitin-dependent protein catabolic process". The terms with low $\mathrm{Ka} / \mathrm{Ks}$ ratio probably represent the most conservative functions, and the terms with high $\mathrm{Ka} / \mathrm{Ks}$ ratio likely represent the rapidly evolving functions between the two species [10,35]. 


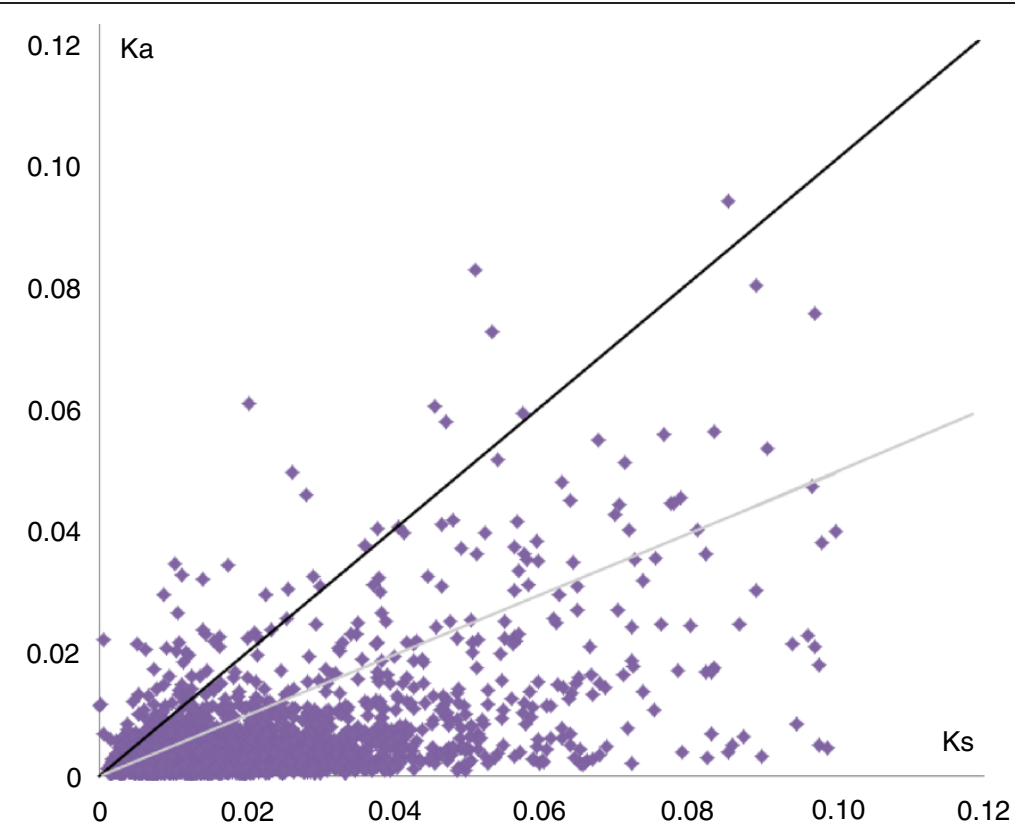

Figure 2 Distribution of Ka and Ks values. Orthologous pairs with Ka/Ks ratio >1 are above the black line; pairs with Ka/Ks ratio between $0.5-1$ are between the black and grey lines.

\section{Functional analysis for candidate genes}

Among the 460 candidate genes $(\mathrm{Ka} / \mathrm{Ks}>0.5)$, 315 were annotated to GO categories (68.5\%). Using the program WEGO [36], we compared the GO category distribution (level 3) of the candidate genes with that of the noncandidate genes $(\mathrm{Ka} / \mathrm{Ks}<0.5)$ (Figure 3). Seven GO terms had a higher percentage among the candidate genes than among the non-candidate genes. Several of these terms were associated with immunity and host defense, such as "immune response" (4.4\% among candidate genes vs. $4.2 \%$ among non-candidate genes), "leukocyte activation" (3.8\% vs. $2.4 \%$ ), and "lymphocyte

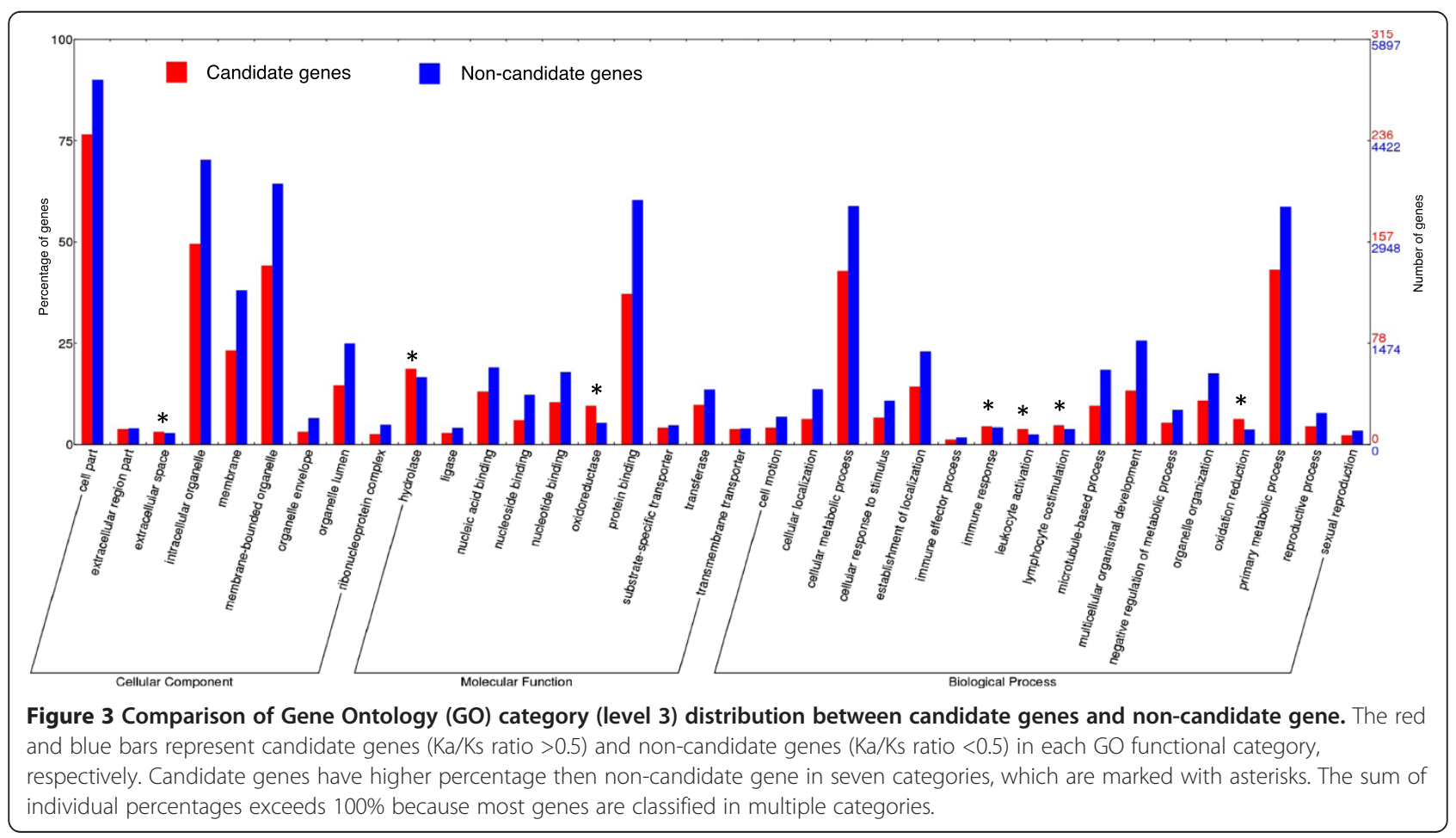


costimulation" (4.8\% vs. $3.8 \%)$. Immune related genes were commonly found under positive selection during speciation processes and in species that do not live at high elevations [35,37]. Therefore, although under positive selection, these genes may not be related to adaptation to a high-elevation environment.

To identify genes that may closely associate to the adaptation to high elevations, we performed a two-step approach to screen all the candidate genes according to their functions. First, we compared our candidate genes to a list proposed by Simonson et al. [20], which encompasses the functions and their associated GO categories for adaptation to high-elevation hypoxia. Two genes associated with "response to hypoxia" and two genes associated with "oxygen binding" were identified from our candidate gene list (Table 2). Second, based on our current understanding on phenotypic adaptation to high elevations of poikilothermic species, we selected three other candidate functions, which are associated with response to ultraviolet rays [11], reactive oxygen species metabolism, and metabolic regulation [12]. Ten additional genes were identified with these candidate functions, which were in the GO categories "response to UV", "glutathione metabolic process", and "ATP catabolic process" (Table 2).

The two candidate genes associated with "oxygen binding", CYP2F1 and CYP4B1, belong to the cytochrome P450 superfamily (CYP). The function of CYP is to catalyze the oxidation of organic substances [38]. The mean $\mathrm{Ka} / \mathrm{Ks}$ ratio of the term "oxygen binding" is 0.2435 , much higher than the mean $\mathrm{Ka} / \mathrm{Ks}$ ratio of all orthologs (0.1452), suggesting a strong overall positive selection on genes in this category. Previous studies of Tibetan people also found that several other CYP genes showed signatures of positive selection related to "oxygen binding" [20]. Our finding suggests a functional (phenotypic) convergent evolution between humans and Rana kukunoris, although the genes behind the functional adaptation are different (but belong to the same gene family).

The gene Ubiquitin-specific protease 1 (USP-1) is also under strong positive selection $(\mathrm{Ka} / \mathrm{Ks}=1.2268)$, and we found a non-synonymous substitution in its His domain of N-terminal, which is likely crucial for its function [39]. USP-1 is a conserved gene that encode an enzyme playing an important role in DNA repair against UV damage $[39,40]$. Thus, USP-1 may be especially important for amphibians to adapt to the high UV radiation environment, since their skin is completely exposed without any protection from fur or scales.

Four genes, GPX4, GSTO2, PRDX4, SCP2, which are associated with response to reactive oxygen species (ROS), have also experienced positive selection (Table 2). The first two genes encode crucial enzymes in glutathione metabolic pathway, which prevent damage to important cellular components by ROS-like peroxides and free radicals [41]. The functions of the latter two genes are related to the functions of peroxisome that are also associated with response to oxidative stress [42,43]. Bickler and Buck [12] proposed that the ability of poikilothermic animals to survive under low temperature and oxygen conditions depends on the ability of avoiding free-radical injury. The four genes identified here are potentially related to the adaptive process in this aspect.

We also identified five metabolism related genes that may be associated with adaptation to high elevations. The MTO-1 and V-ATPase G1 genes are related to ATP synthesis and energy metabolism in the mitochondrion

Table 2 List of fourteen candidate genes with related Gene Ontology (GO) categories

\begin{tabular}{lllll}
\hline Sequence ID & Protein homolog & Ka/Ks ratio & GO category & GO accession \\
\hline Kuku_seq_14688 & Glutathione s-transferase omega 2, GSTO2 & 1.7284 & glutathione metabolic process & GO:0006749 \\
Kuku_seq_38792 & Sterol carrier protein 2, SCP2 & 1.4657 & peroxisome organization & GO:0007031 \\
Kuku_seq_19028 & Ubiquitin-specific protease 1, USP-1 & 1.2268 & response to UV & GO:0009411 \\
Kuku_seq_38041 & Ribosomal L1 domain containing 1,RSL1D1 & 1.1671 & primary metabolic process & GO:0044238 \\
Kuku_seq_19815 & Peroxiredoxin-4, PRDX4 & 1.1484 & response to oxidative stress & GO:0006979 \\
Kuku_seq_9362 & Mitochondrial translation optimization 1, MTO-1 & 1.1363 & mitochondrial tRNA wobble uridine modification & GO:0070899 \\
Kuku_seq_22135 & NADPH oxidase organizer 1, NOXO1 & 1.0432 & NADPH oxidase complex & GO:0043020 \\
Kuku_seq_29724 & Aminoacylase-1, ACY1 & 1.0342 & protein catabolic process & GO:0030163 \\
Kuku_seq_10530 & DNA repair protein xrcc, XRCC & 0.6313 & response to hypoxia & GO:0001666 \\
Kuku_seq_19044 & 4-aminobutyrate aminotransferase, ABAT & 0.5899 & response to hypoxia & GO:0001666 \\
Kuku_seq_19251 & Cytochrome p450 4b1-like, CYP4B1 & 0.5867 & oxygen binding & GO:0019825 \\
Kuku_seq_4377 & Glutathione peroxidase 4, GPX4 & 0.5799 & glutathione metabolic process & GO:0006749 \\
Kuku_seq_29173 & V-type proton ATPase subunit G 1, V-ATPase G1 & 0.5173 & ATP catabolic process & GO:0006200 \\
Kuku_seq_8042 & Cytochrome p450 2f1-like, CYP2F1 & 0.5062 & oxygen binding & GO:0019825 \\
\hline
\end{tabular}

These fourteen genes have $\mathrm{Ka} / \mathrm{Ks}$ ratio greater than 0.5 and are most likely involved in the adaptation to high-elevation environments. 
$[44,45]$; both functions are important in the process of high-elevation adaptation [12]. The ACY1, NOXO1 and RSL1D1genes are related to primary, NADPH and protein metabolism, respectively [46-48]. Functional changes associated with metabolic processes are likely essential for the adaptation to high-elevation environments.

A surprising absence from our candidate gene list is the hemoglobin related genes. Responses to hypoxia in endothermic animal species involve mostly hemoglobin genes and HIF-1 pathways, and we did not observe any of those genes bearing signature of positive selection. The mean $\mathrm{Ka} / \mathrm{Ks}$ ratio (0.1154) of the entire term "response to hypoxia" was lower than average level (0.1452), although two genes (ABAT and XRCC) had $\mathrm{Ka} / \mathrm{Ks}$ ratio greater than 0.5 but less than 1 (Table 2). Some other genes involved in adaptation to high elevations in endothermic species also had a low $\mathrm{Ka} / \mathrm{Ks}$ ratio in our comparison, for example, EPAS1 [20,21] $(\mathrm{Ka} / \mathrm{Ks}=0.1057), \mathrm{ARG} 2[10](\mathrm{Ka} / \mathrm{Ks}=0.1095)$, and EP300 [19] $(\mathrm{Ka} / \mathrm{Ks}=0.1725)$. The gene EGLN1 [19] is an exception in this sense; it has a relatively high $\mathrm{Ka} / \mathrm{Ks}$ ratio of 0.4563, which may deserve further examination. This observation is consistent with our prediction that poikilothermic species may develop different adaptive mechanisms from endotherms.

\section{Future directions}

Our study demonstrated that comparing sequences of orthologous genes between species by de novo transcriptome sequencing offers a fast and cost-effective approach to detect positive selection and to understand adaptive evolution at the genomic level. Further tests are needed to validate these candidate genes that we have identified. Population-level analysis can be applied to these candidate genes to examine if signals of positive selection on these genes are also evident at the population level. Detailed functional research is also needed to illustrate how the positively selected genes promote the fitness of poikilothermic species under the high-elevation environment. Finally, to understand the general genetic mechanisms of adaptation to high elevations of poikilothermic animals, it is necessary to examine other poikilothermic species from various groups. For example, hemoglobin genes, which were thought to play an important role in adaptation to high elevations in endothermic species, were absent from our candidate gene list and the importance of their role remained largely unknown in other poikilothermic species [17]. Studies on a wide range of species are necessary to understand parallel and convergent adaptive evolution to high elevations between poikilotherms and endotherms.

Better and more elaborative tests for positive selection should be explored in order to improve their sensitivity.
$\mathrm{Ka} / \mathrm{Ks}$ ratio can only serve as a preliminary approximate estimator. Functional analysis with pre-defined "potential functions for adaptation", or candidate genes, largely limits the scope of discovery. Only with tests that are solely based on genetic data at a genome-wide scope, it is possible to establish a new 'genotype to phenotype' approach to the study of adaptation, which will complement the traditional 'phenotype to genotype' approach. With this new approach, we can take the great advantage of rapidly accumulating genomic data to reveal novel phenotypes of adaptation.

\section{Conclusions}

By comparing the transcriptomes of two ranid species, one from high and one from low altitudes, we identified 125 protein-coding genes that have experienced strong positive selection $(\mathrm{Ka} / \mathrm{Ks}>1)$, and 335 genes that may also bear signatures of positive selection $(1 \geq \mathrm{Ka} /$ Ks $>0.5$ ). Among them, 14 genes are most likely involved in the adaptive process to high-elevation environments, particularly genes associated with oxygen binding (CYP4B1and CYP2F1), response to UV (USP-1), and repair of free radical injuries (GSTO2, GPX4, PRDX4, and SCP2). Furthermore, we found evidence for convergent evolution between poikilothermic and endothermic species; genes from the CYP superfamily are under positive selection in both humans and amphibians. Yet, we also observed differences between them; hemoglobin related genes are commonly involved in high-elevation adaptation in endotherms, but are absent from our candidate gene list. The genes that display signatures of positive selection will serve as a baseline for further investigations that aim to understand high-elevation adaptation at both molecular and phenotypic levels.

\section{Methods \\ Sample collection}

To include as many expressed genes as possible, we sampled five different types of tissues from six individuals of each species, including liver, heart, skeletal muscle, testicle/ootheca from two males and two females, and the bodies of two tadpoles. Samples of $R$. chensinensis were collected in April, 2011, from the Yaodu Township, Qingchuan County, located on northern Sichuan Basin, China $\left(105.45^{\circ} \mathrm{E}, 32.79^{\circ} \mathrm{N}\right)$ with an altitude of $604 \mathrm{~m}$ above sea level (a.s.l.). Samples of R. kukunoris were collected in April, 2011, from the Zoige County, situated on the eastern margin of the Tibetan Plateau, China $\left(102.90^{\circ} \mathrm{E}, 33.58^{\circ} \mathrm{N}\right)$ with an altitude of 3,358 $\mathrm{m}$ a.s.l. Sample locations are depicted on a map in Additional file 4. Tissue samples for RNA extraction were stored in RNALater (QIAGEN) immediately following euthanasia and dissection. 


\section{cDNA library construction and Illumina sequencing}

RNA extraction, cDNA library construction, and sequencing were performed by BGI (Shenzhen, China). The entire process followed a standardized procedure and was monitored by BGI's Quality Control System. For each species, total RNA was extracted using SV Total RNA Isolation System (Promega) from each tissue sample independently and then mixed with approximately the same quantity. RNAs with poly (A) tail were purified from total RNA by oligonucleotide (dT) magnetic beads and interrupted into short sequences. Subsequently, cDNAs were synthesized and purified with PCR extraction kit (QiaQuick). The cDNA library had an insert size of 200-700 base pair (bp), and paired-end of the library was sequenced with a read length of $90 \mathrm{bp}$ using the Illumina $\mathrm{HiSeq}^{\mathrm{TM}} 2000$ sequencing platform. Output of image data from the sequencer was transformed by base calling into raw sequence data, which formed the raw reads and then stored in the FastQ format.

\section{Data filtration and de novo assembly}

We first cleaned the raw sequence reads by removing the exact duplicates from both sequencing directions. We further trimmed reads by removing adapter sequences, reads with unknown bases call $(\mathrm{N})$ more than $5 \%$, low complexity, and low quality $(<\mathrm{Q} 20)$ using Blat [49]. We also removed reads likely derived from contaminants by comparing with the human and Escherichia coli genomes using Bowtie [50].

De novo assembly of cleaned reads was performed using a combination of various multiple K-mer lengths and coverage cut-off values [26,27]. Using VELVET [51] and OASES [52], five different K-mer lengths (21, 31, 41, 51, and 61$)$ and six coverage cut-off values (2, 3, 6, 10, 15, and 20) were used to produce a total of 30 raw assemblies for each transcriptome. We then used CD-HIT-EST [53] and CAP3 [54] to produce a final assembly by integrating sequence overlaps and eliminating redundancies. All reads were mapped back to the assembled contigs using SOAPALIGNER [55] and SNPs were identified using SOAPSNP [56]. For all variable sites, the base call with the most mapped reads was selected as the consensus using an inhouse Python script.

\section{Gene annotation}

Due to the lack of genomic resources from any Rana species, we combined protein data from seven species covering all major vertebrate groups to create a reference dataset for gene annotation, which includes Anolis carolinensis, Danio rerio, Gallus gallus, Homo sapiens, Mus musculus, Oryzias latipes, and Xenopus tropicalis [57]. All protein data were downloaded from the Ensembl database [58]. Transcripts were annotated to the reference dataset based on the BLAST similarity using blastx with an
E-value cut-off of 1E-5. Transcripts that did not match to any gene in the reference dataset were searched against the non-redundant (NR) protein database of GenBank.

\section{Gene ontology classification}

Gene Ontology [30] categories were used to classify the functions of the transcripts. In order to exclude the interference from alternative splicing of transcripts, we first clustered the transcripts that matched to the same reference gene; then we removed the redundant transcripts and only reserved the longest transcript for each cluster to represent the unique gene. GO classification was performed following the Blast2GO pipelines [59], based on a standard cut-off E-value of $1 \mathrm{E}-3$ and a maximum of 20 hits of the BLAST process, and the default parameters of mapping and annotation procedure.

\section{Identification of putative orthologs}

A reciprocal best hit method was used to identify putative orthologs between the two species with tblastx of BLAST-2.2.26+ and a bit-score threshold of 300 [31]. Subsequently, sequences in each orthologous pair were compared to the combined protein reference to determine their open reading frames (ORF) by blastx. The coding region of both sequences was then extracted and aligned by MUSCLE [60]. Aligned sequences with unexpected stopcodons, ambiguous alignments, and/or shorter than $200 \mathrm{bp}$ were discarded from further analysis. A manual check was also conducted to correct potential errors. All transcripts, including products of potential alternative splicing, were blasted to maximize the chance of finding matches between the two species. If any orthologs that matched to the same reference gene had overlapping annotated region, we removed the shorter sequences to ensure that all orthologous pairs represented unique genes. For contigs that could not be annotated, we also applied the same method to identify orthologous pairs, then used EMBOSS-GETORF to predict the ORF for each sequence [32]. We adopted a strict criterion that only reserved the predicted coding sequence longer than 300 bp of each sequence. The resulting sequence pairs were analysed following the same approach described above.

\section{Test for positive selections}

The ratio of the number of nonsynonymous substitutions per nonsynonymous site (Ka) to the number of synonymous substitutions per synonymous site $(\mathrm{Ks})$ was used to test for positive selection. A $\mathrm{Ka} / \mathrm{Ks}$ ratio less than one indicates purifying selection, while a $\mathrm{Ka} / \mathrm{Ks}$ ratio greater than one provides evidence for positive selection [33]. For its simplicity and applicability, the ratio has been widely used in genome wide comparative studies, despite its limitations. Yang and Bielawski [33] provided a thorough evaluation of the application of $\mathrm{Ka} / \mathrm{Ks}$ for testing selection. 
We employed the software KaKs_CALCULATOR [61] to estimate the $\mathrm{Ka}$, $\mathrm{Ks}$ and $\mathrm{Ka} / \mathrm{Ks}$ ratio of each orthologous pair, and the YN model [62] was used. The sequences with Ks $>0.1$ were excluded to avoid potential paralogs [63]. Based on the GO classification, the unique orthologs were clustered into different functional terms and the mean $\mathrm{Ka} / \mathrm{Ks}$ ratio for each term was also calculated.

\section{Functional analysis for candidate genes}

The orthologs that had $\mathrm{Ka} / \mathrm{Ks}$ ratio above 0.5 were considered as candidate genes [34]. To detect which functions were more likely under positive selection, we compared the GO distribution (level 3) of the candidate genes with distribution of the non-candidate genes $(\mathrm{Ka} / \mathrm{Ks}<0.5)$ by the program WEGO [36]. Furthermore, we performed a two-step approach to identify candidate genes that might have been involved in facilitating the adaptation process to high-elevation environments. First, we examined the list of a priori functional candidate genes and the associated GO categories made by Simonson et al. [20], which represents the functions for adaptation to high elevation hypoxia and was used to screen candidate genes identified from a Tibetan Chinese population [20]. Second, based on previous studies on poikilotherms, we also studied other GO categories and selected the terms potentially associated with response to ultraviolet rays (UV), reactive oxygen species metabolism and metabolic regulation, which may represent other functions that have likely facilitated the adaptation to high elevations $[11,12,64]$.

\section{Availability of supporting data}

The data supporting the results of this article are available in the NCBI Sequence Read Archive (SRA) repository [Accession Number: SRA060325].

\section{Additional files}

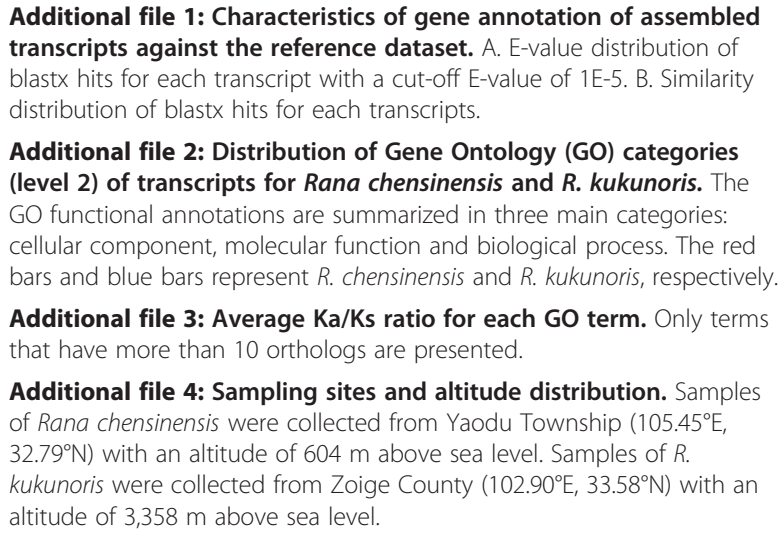

Additional file 4: Sampling sites and altitude distribution. Samples of Rana chensinensis were collected from Yaodu Township $\left(105.45^{\circ} \mathrm{E}\right.$ $32.79^{\circ} \mathrm{N}$ ) with an altitude of $604 \mathrm{~m}$ above sea level. Samples of $R$. kukunoris were collected from Zoige County $\left(102.90^{\circ} \mathrm{E}, 33.58^{\circ} \mathrm{N}\right)$ with an altitude of 3,358 $\mathrm{m}$ above sea level.

\section{Abbreviations}

ACY1: Aminoacylase-1; ARG2: Arginase, type II; bp: Base pair;

CYP2F1: Cytochrome p450 2f1-like; CYP4B1: Cytochrome p450 4b1-like;
CYP: Cytochrome P450 superfamily; EGLN1: Egl nine homolog 1; EP300: E1A binding protein p300; EPAS1: Endothelial PAS domain-containing protein 1; GO: Gene Ontology; GPX4: Glutathione peroxidase 4; GSTO2: Glutathione stransferase omega 2; Mb: Mega base pairs; MTO-1: Mitochondrial translation optimization 1; NOXO1: NADPH oxidase organizer 1; ORF: Open reading frame; PRDX4: Peroxiredoxin-4; ROS: Reactive oxygen species;

RSL1D1: Ribosomal L1 domain containing 1; SCP2: Sterol carrier protein 2; USP-1: Ubiquitin-specific protease 1; UV: Ultraviolet; V-ATPase G1: V-type proton ATPase subunit G 1; XRCC: DNA repair protein xrcc.

\section{Competing interests}

The authors declare that they have no competing interests.

\section{Authors' contributions}

WY carried out most of the data analyses and drafted the manuscript. YQ collected the specimens. KB participated in data analysis and writing. JF conceived the project and finalized the manuscript. All authors contributed to the project design and manuscript preparation. All authors have read and approved the final manuscript.

\section{Authors' information}

WY is a Ph.D. candidate and this project is part of his dissertation work. He is mostly interested in bioinformatics and related genomic work. YQ is a herpetologist and mostly interested in high elevation herpeto-fauna. KB is a post-doctoral fellow who is interested in detecting genome-wide signatures of natural selection using museum historical specimens. JF is an evolutionary biologist particularly interested in evolutionary phenomena around the Tibet Plateau.

\section{Acknowledgements}

We would like to thank D. Hu for field assistance. J. P. Bogart, Q. Dai, B. Lu, T. F. Mitterboeck, and C. Rochus provided many valuable comments on an early version of this manuscript. This project is funded by a Chinese Academy of Sciences Knowledge Innovation Program grant (Y1C2021203) and an NSERC discovery grant (Canada) to JF.

\section{Author details}

${ }^{1}$ Chengdu Institute of Biology, Chinese Academy of Sciences, Chengdu 610041, China. ${ }^{2}$ Graduate University of Chinese Academy of Sciences, Beijing 100049, China. ${ }^{3}$ Museum of Vertebrate Zoology, University of California, Berkeley, CA 94720-3160, USA. ${ }^{4}$ Department of Integrative Biology, University of Guelph, Guelph, Ontario N1G 2W1, Canada.

Received: 15 August 2012 Accepted: 30 October 2012

Published: 1 November 2012

\section{References}

1. Rose MR: Adaptation. In Encyclopedia of Biodiversity. Edited by Levin RA. San Diego: Academic Press; 2001:17-23.

2. Smith NGC, Eyre-Walker A: Adaptive protein evolution in Drosophila. Nature 2002, 415:1022-1024.

3. Zhu M, Zhao S: Candidate gene identification approach: progress and challenges. Int J Biol Sci 2007, 3:420-427.

4. Huynen MA BP: Measuring genome evolution. Proc Natl Acad Sci USA 1998, 95:5849-5856

5. Li R, Fan W, Tian G, Zhu H, He L, Cai J, Huang Q, Cai Q, Li B, Bai Y, Zhang Z, Zhang Y, Wang W, Li J, Wei F, Li H, Jian M, Li J, Zhang Z, Nielsen R, Li D, Gu W, Yang Z, Xuan Z, Ryder OA, Leung FC, Zhou Y, Cao J, Sun X, Fu Y, et al: The sequence and de novo assembly of the giant panda genome. Nature 2009, 463:311-317.

6. Bonasio R, Zhang G, Ye C, Mutti NS, Fang X, Qin N, Donahue G, Yang P, Li Q, Li C, et al: Genomic Comparison of the Ants Camponotus floridanus and Harpegnathos saltator. Science 2010, 329:1068-1071.

7. Cheviron ZA, Brumfield RT: Genomic insights into adaptation to highaltitude environments. Heredity 2011, 108:354-361.

8. Wilson MJ, Julian CG, Roach RC: Genomic analysis of high-altitude adaptation: innovations and implications. Curr Sport Med Rep 2011 10:59-61.

9. Scheinfeldt LB, Soi S, Thompson S, Ranciaro A, Meskel DW, Beggs W, Lambert C, Jarvis JP, Abate D, Belay G, Tishkoff SA: Genetic adaptation to high altitude in the Ethiopian highlands. Genome Biol 2012, 13:R1. 
10. Qiu Q, Zhang G, Ma T, Qian W, Wang J, Ye Z, Cao C, Hu Q, Kim J, Larkin DM, Auvil L, Capitanu B, Ma J, Lewin HA, Qian X, Lang Y, Zhou R, Wang L, Wang K, Xia J, Liao S, Pan S, Lu X, Hou H, Wang Y, Zang X, Yin Y, Ma H, Zhang J, Wang $Z$, et al: The yak genome and adaptation to life at high altitude. Nat Genet 2012, 44:946-949.

11. Blumthaler M, Ambach W, Ellinger R: Increase in solar UV radiation with altitude. J Photoch Photobio B 1997, 39:130-134.

12. Bickler PE, Buck LT: Hypoxia tolerance in reptiles, amphibians, and fishes: life with variable oxygen availability. Annu Rev Physiol 2007, 69:145-170.

13. Durmowicz AG, Hofmeister S, Kadyraliev T, Aldashev AA, Stenmark KR: Functional and structural adaptation of the Yak pulmonary circulation to residence at high altitude. J App/ Physio/ 1993, 74:2276-2285.

14. Savourey G, Garcia N, Besnard Y, Guinet A, Hanniquet AM, Bittel J: Preadaptation, adaptation and de-adaptation to high altitude in humans: cardio-ventilatory and haematological changes. Eur J Appl Physiol Occup Physiol 1996, 73:529-535

15. Milsom WK, Scott G: Respiratory adaptations in the high flying barheaded goose. Comp Biochem Physiol C: Toxicol Pharmacol 2008, 148:460.

16. Storz JF, Sabatino SJ, Hoffmann FG, Gering EJ, Moriyama H, Ferrand N, Monteiro B, Nachman MW: The molecular basis of high-altitude adaptation in deer mice. PLOS Genet 2007, 3:e45.

17. Weber RE: High-altitude adaptations in vertebrate hemoglobins. Respir Physiolo Neurobiol 2007, 158:132-142.

18. Yu L, Wang $X$, Ting N, Zhang Y: Mitogenomic analysis of Chinese snubnosed monkeys: evidence of positive selection in NADH dehydrogenase genes in high-altitude adaptation. Mitochondrion 2011, 11:497-503.

19. Peng Y, Yang Z, Zhang H, Cui C, Qi X, Luo X, Tao X, Wu T, Ouzhuluobu B, Ciwangsangbu D, Chen H, Shi H, Su B: Genetic variations in Tibetan populations and high-altitude adaptation at the Himalayas. Mol Biol Evol 2010, 28:1075-1081.

20. Simonson TS, Yang Y, Huff CD, Yun H, Qin G, Witherspoon DJ, Bai Z, Lorenzo FR, Xing J, Jorde LB: Genetic evidence for high-altitude adaptation in Tibet. Science 2010, 329:72-75.


Vinckenbosch N, Korneliussen TS, Zheng H, Liu T, He W, Li K, Luo R, Nie X, Wu H, Zhao M, Cao H, Zou J, Shan Y, Li S, Yang Q, Asan, Ni P, Tian G, Xu J, Liu $X$, Jiang $T$, Wu R, et al: Sequencing of 50 human exomes reveals adaptation to high altitude. Science 2010, 329:75-78.

22. Fei L, Hu S, Ye C, Huang Y: Fauna Sinica, Amphibia, Volume 2. Beijing: Science Press; 2009.

23. Zhou W-w, Wen Y, Fu J, Xu Y-b, Jin J-q, Ding LI, Min M-q, Che J, Zhang Y-p: Speciation in the Rana chensinensis species complex and its relationship to the uplift of the Qinghai-Tibetan Plateau. Mol Ecol 2012, 21:960-973.

24. Li S: Cytotaxonomy of Amphibians in China. Beijing: Science Press; 2007.

25. Vinogradov AE: Genome size and GC-percent in vertebrates as determined by flow cytometry: the triangular relationship. Cytometry 1998, 31:100-109.

26. Surget-Groba Y, Montoya-Burgos Jl: Optimization of de novo transcriptome assembly from next-generation sequencing data. Genome Res 2010, 20:1432-1440.

27. Gruenheit N, Deusch O, Esser C, Becker M, Voelckel C, Lockhart PJ: Cutoffs and k-mers: implications from a transcriptome study in allopolyploid plants. BMC Genomics 2012, 13:92.

28. Robertson G, Schein J, Chiu R, Corbett R, Field M, Jackman SD, Mungall K, Lee S, Okada HM, Qian JQ, Griffith M, Raymond A, Thiessen N, Cezard T, Butterfield YS, Newsome R, Chan SK, She R, Varhol R, Kamoh B, Prabhu AL, Tam A, Zhao Y, Moore RA, Hirst M, Marra MA, Jones SJM, Hoodless PA, Birol I: De novo assembly and analysis of RNA-seq data. Nat Methods 2010, 7:909-912.

29. Garg R, Patel RK, Tyagi AK, Jain M: De novo assembly of chickpea transcriptome using short reads for gene discovery and marker identification. DNA Res 2011, 18:53-63.

30. Ashburner M, Ball CA, Blake JA, Botstein D, Butler H, Cherry JM, Davis AP, Dolinski K, Dwight SS, Eppig JT: Gene Ontology: tool for the unification of biology. Nat Genet 2000, 25:25-29.

31. Altenhoff AM, Dessimoz C: Phylogenetic and functional assessment of orthologs inference projects and methods. PLoS Comput Biol 2009, 5:e1000262.

32. Rice $\mathrm{P}$, Longden I, Bleasby A: EMBOSS: the European molecular biology open software suite. Trends Genet 2000, 16:276-277.

33. Yang Z, Bielawski JP: Statistical methods for detecting molecular adaptation. Trends Ecol Evol 2000, 15:496-503.
34. Swanson WJ: Evolutionary expressed sequence tag analysis of Drosophila female reproductive tracts identifies genes subjected to positive selection. Genetics 2004, 168:1457-1465.

35. The Chimpanzee Sequencing and Analysis Consortium: Initial sequence of the chimpanzee genome and comparison with the human genome. Nature 2005, 437:69-87.

36. Ye J, Fang L, Zheng H, Zhang Y, Chen J, Zhang Z, Wang J, Li S, Li R, Bolund L: WEGO: a web tool for plotting GO annotations. Nucleic Acids Res 2006, 34:W293-W297

37. Li W, Saunders MA: The chimpanzee and us. Nature 2005, 437:50-51.

38. Danielson P: The cytochrome P450 superfamily: biochemistry, evolution and drug metabolism in humans. Curr Drug Metab 2002, 3:561-597.

39. Cotto-Rios XM, Jones MJK, Busino L, Pagano M, Huang T: APC/CCdh1dependent proteolysis of USP1 regulates the response to UV-mediated DNA damage. J Cell Biol 2011, 194:177-186.

40. Fujiwara T, Saito A, Suzuki M, Shinomiya H, Suzuki T, Takahashi E, Tanigami A, Ichiyama A, Chung CH, Nakamura Y: Identification and chromosomal assignment of USP1, a novel gene encoding a human ubiquitin-specific protease. Genomics 1998, 54:155-158.

41. Pompella A, Visvikis A, Paolicchi A, Tata VD, Casini AF: The changing faces of glutathione, a cellular protagonist. Biochem Pharmacol 2003, 66:1499-1503

42. Giguere $P$, Turcotte $M$, Hamelin E, Parent A, Brisson J, Laroche G, Labrecque $P$, Dupuis G, Parent J: Peroxiredoxin-4 interacts with and regulates the thromboxane A2 receptor. FEBS Lett 2007, 581:3863-3868.

43. Offenberg HH, Schalk JAC, Meuwissen RL, van Aalderen M, Kester HA, Dietrich AJJ, Heyting C: SCP2: a major protein component of the axial elements of synaptonemal complexes of the rat. Nucleic Acids Res 1998, 26:2572-2579

44. Wang X, Yan Q, Guan M-X: Mutation in MTO1 involved in tRNA modification impairs mitochondrial RNA metabolism in the yeast Saccharomyces cerevisiae. Mitochondrion 2009, 9:180-185.

45. Beyenbach KW, Wieczorek H: The V-type H+ ATPase: molecular structure and function, physiological roles and regulation. J Exp Biol 2006, 209:577-589.

46. Cheng G: Nox3 Regulation by NOX01, p47phox, and p67phox. J Biol Chem 2004, 279:34250-34255.

47. Sass JO, Mohr V, Olbrich H, Engelke U, Horvath J, Fliegauf M, Loges NT, Schweitzer-Krantz S, Moebus R, Weiler P: Mutations in ACY1, the gene encoding aminoacylase 1, cause a novel inborn error of metabolism. Am J Hum Genet 2006, 78:401-409.

48. Ma L, Chang N, Guo S, Li Q, Zhang Z, Wang W, Tong T: CSIG inhibits PTEN translation in replicative senescence. Mol Cell Biol 2008, 28:6290-6301.

49. Kent WJ: BLAT-the BLAST-like alignment tool. Genome Res 2002, 12:656-664.

50. Langmead B, Trapnell C, Pop M, Salzberg SL: Ultrafast and memoryefficient alignment of short DNA sequences to the human genome. Genome Biol 2009, 10:R25.

51. Zerbino DR, Birney E: Velvet: Algorithms for de novo short read assembly using de Bruijn graphs. Genome Res 2008, 18:821-829.

52. Schulz MH, Zerbino DR, Vingron M, Birney E: Oases: Robust de novo RNAseq assembly across the dynamic range of expression levels. Bioinformatics 2012, 28:1086-1092.

53. Li W, Godzik A: Cd-hit: a fast program for clustering and comparing large sets of protein or nucleotide sequences. Bioinformatics 2006, 22:1658-1659.

54. Huang $X$, Madan A: CAP3: a DNA sequence assembly program. Genome Res 1999, 9:868-877.

55. Li R, Yu C, Li Y, Lam TW, Yiu SM, Kristiansen K, Wang J: SOAP2: an improved ultrafast tool for short read alignment. Bioinformatics 2009, 25:1966-1967.

56. Li R, Li Y, Fang X, Yang H, Wang J, Kristiansen K: SNP detection for massively parallel whole-genome resequencing. Genome Res 2009, 19:1124-1132.

57. Castoe TA, Fox SE, de Koning AP J, Poole AW, Daza JM, Smith EN, Mockler TC, Secor SM, Pollock DD: A multi-organ transcriptome resource for the Burmese Python (Python molurus bivittatus). BMC Res Notes 2011, 4:310.

58. Hubbard TJP, Aken B, Beal K, Ballester B, Caccamo M, Chen Y, Clarke L, Coates G, Cunningham F, Cutts T: Ensembl 2007. Nucleic Acids Res 2007, 35:D610-D617.

59. Conesa A, Gotz S, Garcia-Gomez JM, Terol J, Talon M, Robles M: Blast2GO: a universal tool for annotation, visualization and analysis in functional genomics research. Bioinformatics 2005, 21:3674-3676. 
60. Edgar RC: MUSCLE: multiple sequence alignment with high accuracy and high throughput. Nucleic Acids Res 2004, 32:1792-1797.

61. Zhang Z, Li J, Zhao XQ, Wang J, Wong GKS, Yu J: KaKs_Calculator: calculating $\mathrm{Ka}$ and $\mathrm{Ks}$ through model selection and model averaging. Genomics Proteomics \& Bioinfor 2006, 4:259-263.

62. Yang Z, Nielsen R: Estimating synonymous and nonsynonymous substitution rates under realistic evolutionary models. Mol Biol Evol 2000, 17:32-43.

63. Elmer KR, Fan S, Gunter HM, Jones JC, Boekhoff S, Kuraku S, Meyer A: Rapid evolution and selection inferred from the transcriptomes of sympatric crater lake cichlid fishes. Mol Ecol 2010, 19:197-211.

64. Yang H, Wang X, Liu X, Wu J, Liu C, Gong W, Zhao Z, Hong J, Lin D, Wang $Y$, Lai R: Antioxidant peptidomics reveals novel skin antioxidant system. Mol Cell Proteomics 2008, 8:571-583.

doi:10.1186/1471-2164-13-588

Cite this article as: Yang et al:: Toward understanding the genetic basis of adaptation to high-elevation life in poikilothermic species: A comparative transcriptomic analysis of two ranid frogs, Rana chensinensis and R. kukunoris. BMC Genomics 2012 13:588.

\section{Submit your next manuscript to BioMed Central and take full advantage of:}

- Convenient online submission

- Thorough peer review

- No space constraints or color figure charges

- Immediate publication on acceptance

- Inclusion in PubMed, CAS, Scopus and Google Scholar

- Research which is freely available for redistribution 